\title{
The Role of Ninjurin 1 and Its Impact beyond the Nervous System
}

\author{
Ke Liu Yong Wang Hongge Li \\ Department of Neurology, Union Hospital, Tongji Medical College, Huazhong University of Science and Technology, \\ Wuhan, China
}

\section{Keywords}

Ninjurin 1 - Nerve injury · Inflammation · Tumorigenesis .

Tissue homeostasis

\begin{abstract}
Ninjurin1 (Ninj1) is a double-transmembrane cell surface protein that could promote nerve regeneration in the process of the peripheral nervous system injury and repairment. Nonetheless, the accurate function of Ninj1 in the central nervous system and outside the nervous system is not completely clear. According to the recent studies, we found that Ninj1 is also aberrantly expressed in various pathophysiological processes in vivo, including inflammation, tumorigenesis, and vascular, bone, and muscle homeostasis. These findings suggest that Ninj1 may play an influential role during these pathophysiological processes. Our review summarizes the diverse roles of Ninj1 in multiple pathophysiological processes inside and outside the nervous system. Ninj1 should be considered as an important and novel therapeutic target in certain diseases, such as inflammatory diseases and ischemic diseases. Our study provided a better understanding of Ninj1 in different pathophysiological processes and thereby provided the theoretical support for further research.
\end{abstract}

(c) 2021 S. Karger AG, Basel

\section{Introduction}

Ninjurin family proteins (ninjurins) are a group of double-transmembrane proteins, whose expression is significantly upregulated both in Schwann cells and dorsal root ganglion (DRG) neurons following nerve injury $[1,2]$. And, it has been confirmed that ninjurins are also a class of cell surface molecules that mediate cell-to-cell adhesion via homophilic binding in vitro $[1,2]$. Until now, ninjurins mainly contain 2 homologous molecules in mammals, Ninjurin 1 (Ninj1) and Ninjurin2 (Ninj2), located on different chromosomes $[3,4]$. Interestingly, Ninj1 and Ninj2 share 2 conserved transmembrane hydrophobic regions, whereas they do not bind to each other due to their unique homophilic adhesion motifs [2, 4]. Ninj1 consists of 2 hydrophobic transmembrane regions (amino acids $72-100$ and 118139), an intracellular region, and 2 extracellular regions at the $\mathrm{N}$ - and C-termini $[1,5]$. Furthermore, a 12-amino acid residue domain consisting of a tryptophan residue and a cluster of arginine residues, from $\mathrm{Pro}^{26}$ to $\mathrm{Asn}^{37}$, exists in the N-terminal extracellular region of Ninj1, which is also a region identified as having homophilic adhesion properties [2]. There is also an N-glycosylation site in the extracellular region of the $\mathrm{N}$-terminus of Ninj1 at $\mathrm{Asn}^{60}[1,5]$. The $\mathrm{N}$-glycosylation modification of $\mathrm{Asn}^{60}$ is not only one of the important posttranslational modifications of Ninj1, but

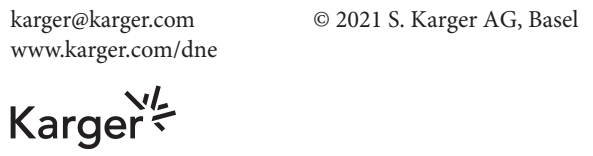




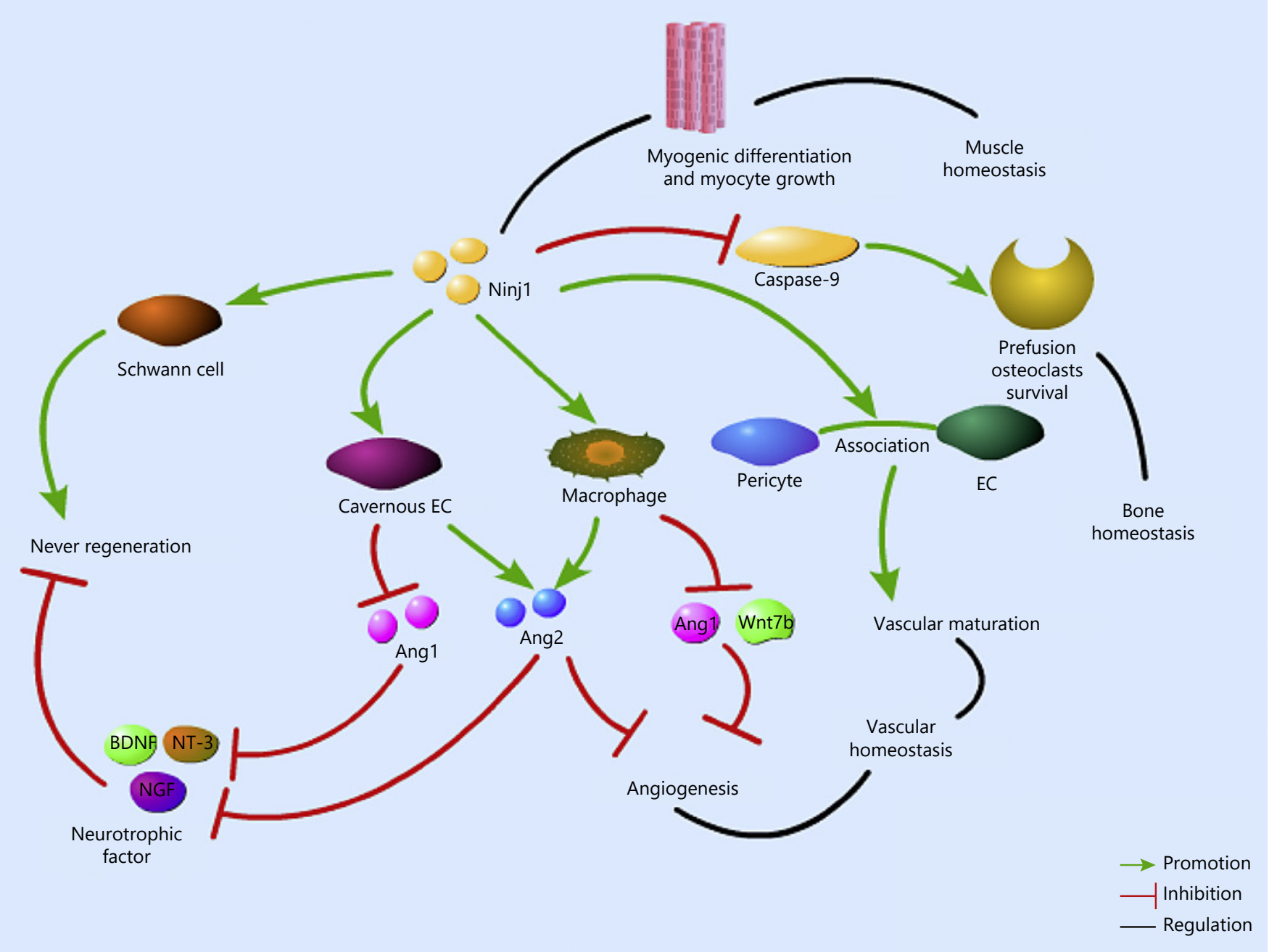

Fig. 1. Proposed mechanisms of Ninj1 function during the process of nerve regeneration as well as vascular, bone, and muscle homeostasis. preOCs, prefusion osteoclasts; EC, endothelial cell; PC, pericyte.

also a prerequisite for Ninj1 to form a homologous protein complex [5].

In addition to being expressed in the injured peripheral nervous system (PNS), Ninj1 exists in various tissues of the body as well, and Ninj1 is chiefly expressed in embryonic and adult epithelial tissues, such as the liver, kidney, thymus, uterus, adrenal gland, and retina [2]. This indicates that except for its role in promoting nerve regeneration, Ninj1 may also participate in the organogenesis of various tissues, similar to some adhesion molecules, such as neural cell adhesion molecule or L1 cell adhesion molecule [1, 6-8]. In recent years, with the continuous deepening of research on Ninj1, it has been found that Ninj1 is involved in various pathophysiological processes in vivo, including nerve regeneration, in- flammation inside and outside the nervous system, tumorigenesis, and tissue homeostasis. Thus, Ninj1 is likely to become a novel therapeutic target for many diseases. In this review, we will summarize the role and mechanism of Ninj1 in the aforementioned pathophysiological processes, discuss the contradictions, and propose the direction for further research of Ninj1. The mechanisms of the Ninj1 function are shown in Figures 1 and 2.

\section{Roles of Ninjurin 1 inside the Nervous System}

\section{Ninjurin1 and PNS}

One of the most obvious differences between the PNS and the central nervous system (CNS) is the ability to re- 


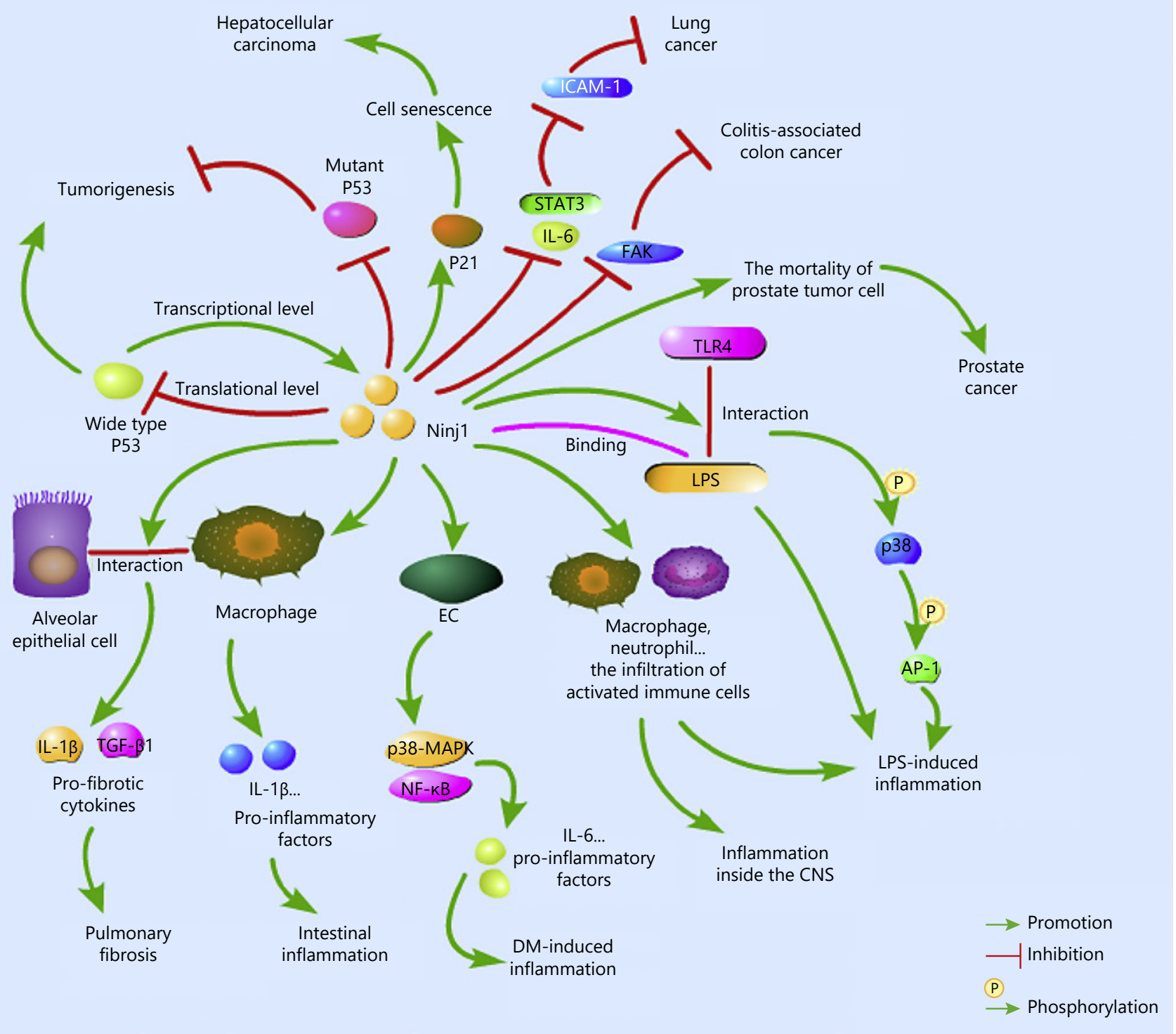

Fig. 2. Proposed mechanisms of Ninj1 function during the process of inflammation and tumorigenesis. WT, wild type; LPS, lipopolysaccharide; EC, endothelial cell; CNS, central nervous system; DM, diabetes mellitus.

generate axons after nerve injury [1]. Ninj1 is increased significantly in Schwann cells, which are the main medium in triggering much of what happens throughout the Wallerian degeneration $[9,10]$, in response to rat sciatic nerve injury $[1,2,11]$. Ninj1 is also upregulated in DRG neurons and is then anterogradely transported to the injured site [1]. By the way of coculture experiments with DRG neurons and Ninj1-expressing Chinese hamster ovary cells, Araki et al. [1] demonstrated that Ninj1 can promote DRG neurons neurite outgrowth in vitro. Sub- sequently, they further confirmed that residues essential for Ninj1-mediated adhesion are also important for Ninj1-promoted neurite outgrowth [1]. In other words, Ninj1-mediated intercellular homophilic adhesion is responsible for Ninj1-promoted neurite outgrowth. This similar effect is also seen in other cell adhesion molecules (CAMs), such as L1 and N-cadherin [12, 13]. Similarly, Jerregard et al. [14] also demonstrated that Ninj1 can promote the survival of neurons and the growth of neurites via coculture experiments of skin-derived fibroblast-like 
cells and DRG neurons in vitro. Furthermore, Tomita et al. [15] further confirmed that Ninj1 is crucial for peripheral nerve regeneration in vivo through the use of a sciatic nerve crush model in an $\mathrm{NG}_{2}^{+}$-specific Ninj1 gene knockout mouse. Overall, all studies mentioned above illustrate the positive role of Ninj1 during neural regeneration after traumatic injury to PNS.

As an adhesion molecule on the cell surface, Ninj1 is not only involved in the pathophysiological process after traumatic nerve injury in the PNS, but also closely related to nontraumatic nerve injury in the PNS, such as leprosy. Leprosy, also known as Hansen's disease, is a chronic infectious disease caused by Mycobacterium leprosy infection and is also an infectious degenerative disease of the PNS $[16,17]$. And, due to its disability and deformity caused by peripheral neuropathy, leprosy is the main cause of nontraumatic peripheral neuropathy $[17,18]$. The gene of Ninj1, NINJ1, contains a nonsynonymous A-C reversal at its third exon, resulting in a change in the amino acid at position 110 (asp110ala) of the protein [19]. Subsequently, Cardoso et al. [19] found that a single nucleotide polymorphism in the NINJ1 gene, asp100ala, was related to the disability grade of leprosy patients through a case-control study of 1,123 individuals. Moreover, further research found that patients carrying the ala 110 allele were more likely to develop nerve damage because they produce lower levels of Ninj1 [19]. After that, Graca et al. [20] reinforced the abovementioned observations with a case-control study of 218 people that leprosy patients carrying the $\mathrm{C}$ allele (ala110) were more likely to develop nerve damage.

In summary, based on the studies mentioned above, it can be concluded that Ninj1 plays an important role in neural regeneration after traumatic or nontraumatic PNS injury. However, interestingly, Ninj1 may be involved in the pathogenesis of endometriosis and adenomyosis inherent pain symptoms due to its role in promoting neurite outgrowth [21]. Therefore, it can be seen that the role of Ninj1 promoting neurite outgrowth is not all beneficial to the human body.

\section{Ninjurin1 and CNS}

Ninj1 is expressed at low levels in the brain of normal adult rats and mainly expressed in 3 major parts of the brain, namely, the meninges, choroid plexus, and parenchymal space around blood vessels [22], which are also main ways for blood-derived immune cell infiltration into the CNS [23]. As we all know, the most important step is to recruit immune cells to cross the blood-brain barrier (BBB) endothelial cells (ECs) to the lesion part during the pathological process of CNS inflammatory diseases [24]. Increasing evidence indicated that the Ninj1 expression is upregulated on the surface of some activated immune cells, including macrophages/monocytes, neutrophils, T lymphocytes, and inflamed vascular ECs in certain CNS inflammatory diseases, such as multiple sclerosis and its rodent model of experimental autoimmune encephalomyelitis (EAE) and ischemic stroke [22, 25-28]. And, it also has been proved that Ninj1 can take part in the transmigration of immune cells across the BBB-ECs via mediating the cell-cell homophilic adhesion in vitro $[22,26]$. Furthermore, Ifergan et al. [26] found that Ninj1 blockade, by Ninj1-blocking antibody or Ninj126-37 blocking peptide, alleviated clinical symptoms of the mouse EAE model through reducing its demyelination and immune cell infiltration [26]. And, Ahn et al. [25] also reached similar conclusions in the EAE model of Ninj1-knockout mice. Moreover, they still found that Ninj1 could promote the immune cells across the EC monolayer by the way of facilitating Rac1-dependent membrane protrusion formation and dynamics [29].

In addition to playing a proinflammatory role in multiple sclerosis and EAE, Ninj1 may also be involved in neutrophil infiltration and the aggravation of brain injury in the postischemic state according to the results obtained by Lee et al. [28] in the middle cerebral artery occlusion (MCAO) model of the rat. And, they also demonstrated that intranasal administration of Ninj1 small interfering RNA or a dodecamer peptide harboring Ninj1 $\mathrm{N}$-terminal adhesion motif (Pro26 Asn37) played a role of neuroprotection and anti-inflammation via improving neurological deficits and motor impairment, which is associated with suppressing acute neutrophil infiltration in the rat MCAO model [30]. Furthermore, on the other hand, Le et al. [31] found obsessive-compulsive disorderlike behaviors in Ninj1-knockout mice, including repetitive- and anxiety-like behaviors without cognitive and social impairments. However, the reasons for the above phenotypes are related to the alterations of synaptic transmission in the thalamic neurons as well as a reduced number of functional synapses and glutamatergic dysfunctions in Ninj1-knockout mice [31].

Taken together with the conclusions of the abovementioned studies, the role of Ninj1 in the CNS can be divided into 2 aspects. On the one hand, Ninj1 is involved in the pathogenesis of the CNS inflammatory diseases via meditating leukocyte-endothelium adhesion in the early stage and protrusive functions during diapedesis in the later stage [29]. On the other hand, Ninj1 could 
participate in neuropsychiatric disorders linking with impairments of repetitive and anxiety behaviors via regulating the synaptic transmission in thalamic neurons and dysregulation of glutamate signaling [31]. Nonetheless, there is no evidence that Ninj1 can promote the repair process after CNS injury, just like Ninj1's role in PNS injury.

\section{Roles of Ninjurin 1 outside the Nervous System}

The Role of Ninjurin1 in Inflammatory Diseases

Ninj1 Is Involved in the Pathogenesis of LPS-Induced Inflammation

As mentioned earlier, Ninj1 is mainly involved in the occurrence and development of inflammatory diseases of the CNS by mediating myeloid cells through the BBB. Given its important role in inflammatory diseases of the CNS, the role of Ninj1 in inflammation outside the nervous system deserves further research. Accordingly, through the study of septic mice, Jennewein et al. [32] found that the expression of Ninj1 is enhanced by inflammatory stimuli. And, further research found that Ninj1 promotes inflammatory response not only by mediating leukocyte infiltration but also by mediating TLR4 signaling via p38 and subsequent activator protein-1 activation [32]. Furthermore, functional inhibition of Ninj1 by the peptide Ninj126-37, whose function has been assessed in the MCAO model [30], not only reduced cytokine plasma levels, such as TNF- $\alpha$, IL-6, and IL-10, and therefore systemic inflammation, but also alleviated the organ damage including the lung and liver in the cecal ligation and puncture model of mice [32].

On the other hand, Shin et al. [33] demonstrated that Ninj1 can also directly bind to the lipid moiety of lipopolysaccharide (LPS) to regulate LPS-induced inflammation in an LPS-induced in vitro inflammation model. Of note, the region of Ninj1, which is responsible for LPS binding, is not the Ninj1 amino acid 26-37, the homophilic binding domain, but the Ninj1 amino acid 81-100, which belongs to the first transmembrane domain [33]. More importantly, the intervention of the binding region between Ninj1 and LPS can precisely regulate the LPSinduced inflammation without affecting the adhesion function of Ninj1.

Ninj1 Is Involved in the Pathogenesis of Intestinal Inflammation and Pulmonary Fibrosis

To our knowledge, several pieces of research elucidate that abnormal activation of proinflammatory macro- phages could lead to intestinal inflammation [34, 35]. Concomitantly, Jung et al. [36] proclaimed that Ninj1 expression was significantly enhanced in the dextran sodium sulfate-induced mice intestinal inflammation models, especially on the surface of macrophages. More importantly, Ninj1-knockout mice exhibited mild clinical phenotype and pathological lesions than wild-type (WT) mice during experimental colitis. Furthermore, Jung et al. [36] concluded that Ninj1 contributes to the activation of macrophages, thereby promoting the secretion of proinflammatory factors and chemokines, such as IL- $1 \beta$, IL-6, and CCL2, via the TLR4-protein Kinase $C \delta / \theta$ signaling pathway during the development of colitis in vivo [36]. Notably, unlike its role in the CNS, Ninj1 does not promote the infiltration of macrophages into the inflammatory lesion site, but rather promotes the activation of macrophages under the intestinal inflammatory conditions [36].

Similarly, some reports indicated that macrophages are a major and important inflammatory cell type to promote fibrosis formation in the pathogenesis of pulmonary fibrosis [37-39], which is a chronic inflammatory process $[40,41]$, and the expression of Ninj1 is also increased on the surface of macrophages in the development of pulmonary fibrosis [42]. Furthermore, Ninj1 is involved in developing pulmonary fibrosis by enhancing contact-dependent interaction between macrophages and alveolar epithelial cells, resulting in the promotion of macrophage activation [42]. However, Ninj1 does not alter the migration capacity of macrophages during the development of pulmonary fibrosis [42]. Consistent with the aforementioned result, Ninj1-knockout mice exhibited mild inflammation and fibrotic lung tissue phenotype, and the expression of proinflammatory mediators and profibrotic cytokines, such as IL- $1 \beta$ and TGF- $\beta 1$, was significantly decreasing as well in the bleomycin-induced pulmonary fibrosis model [42].

Ninj1 Is Involved in the Pathogenesis of Diabetic Endothelial Dysfunction

It is well documented that diabetes mellitus (DM), considered as a low-grade inflammatory disease, is one of the risk factors for endothelial dysfunction [43]. Furthermore, experimental and animal studies have demonstrated that Ninj1 expression was upregulated not only in ECs of type 2 diabetic mice and high-glucose (HG) cultured human umbilical vein ECs but also in ECs of clinical specimens for diabetics [44]. Moreover, in gain- and loss-of function experiments, Wang et al. [44] elucidated that Ninj1 can facilitate the expression of proinflammatory 
genes, such as ICAM-1, VCAM-1, IL-6, and MCP-1, via modulating p38-MAPK and NF- $\mathrm{KB}$ pathways, and the formation of reactive oxygen species under HG condition. On the other hand, Ninj1 also can aggravate endothelial apoptosis not only by enhancing the activation of inflammation-related caspase-3, but also by attenuating the expression of $\mathrm{Bcl}-2$ via mediating the inhibition of the PI3K/Akt signal pathway under HG condition [44]. Besides, Ninj1 aggravates HG-induced endothelial dysfunction by reducing the activation of endothelial nitric oxide synthase, one of the protective factors of the cardiovascular system, via the PI3K/Akt pathway as well [44]. Moreover, Yin et al. [45] also observed a similar phenomenon. They found that Ninj1 was significantly increased in cavernous ECs of diabetic mice with erectile dysfunction (ED). Furthermore, Ninj1 could inhibit the phosphorylation of Akt and endothelial nitric oxide synthase by regulating the angiopoietin-1 (Ang1)/angiopoietin-2 (Ang2) ratio to reduce the survival and regeneration of ECs, whereas Ninj1-neutralizing antibody could reverse this process and thus facilitate the survival and regeneration of ECs [46].

\section{The Role of Ninjurin1 in Cancer Progression}

Ninj1 Is Involved in Tumorigenesis by Regulating

p53 Activity via the p53-Ninj1 Feedback Loop

The tumor suppressor protein p53, often known as the "guardian of the genome," plays a crucial role in tumor suppression by modulating cell cycle arrest, apoptosis, and senescence [47-49]. And, Ninj1 was identified as a p53 target gene by a DNA microarray assay [50]. Specifically, p53 can transcriptionally elevate the expression of Ninj1 via directly binding to the p53-responsive element in the promoter, but the silencing of Ninj1 can translationally enhance p53 expression in return [51]. Additionally, functional inhibition of Ninj1 suppresses cell proliferation, but enhances apoptosis and premature senescence in a p53-dependent way [51]. Furthermore, given that both Ninj1 and p53 play a pivotal role in ionizing radiation response [52, 53], Cho et al. [51] found that Ninj1 deficiency also upregulates ionizing radiation-induced lethality in parallel with enhancive p53 expression in the thymus. On the other hand, it is notable that depending on p53 genetic status, Ninj 1 exerts 2 opposing effects on tumorigenesis [54]. Specifically, Ninj1 deficiency promotes cell proliferation and migration in cells carrying a mutant p53, but suppresses cell growth and migration in cells carrying a WT p53 [54].
Ninj1 Is Involved in Tumorigenesis by Modulating

Cell Motility

As we all know, cell motility, which is one of the main distinctions between benign and malignant tumors, plays an important role in tumor cell invasion and migration. Thus far, several investigations have concentrated on the potential role of Ninj1 in cell motility. First of all, Jang et al. [55] elucidated that Ninj1 deficiency increased the lung cancer cell motility by increasing the expression of ICAM-1 via promoting the IL-6/STAT3 signaling pathway in vitro. ICAM-1 has been reported to notably enhance cell migration and invasion in several tumor cells [56-61]. In line with this observation, inhibition of Ninj1 enhanced the metastatic potential of lung tumor cells and the number and sizes of tumor nodules without affecting tumor growth in an experimental lung metastasis model in vivo [55]. Furthermore, Woo et al. [62] demonstrated that Ninj1 overexpression could decrease the growth of colorectal tumors by suppressing the infiltration of tumor-associated macrophages via suppression of focal adhesion kinase signaling pathways in a mouse model of colitis-associated colon cancer. Tumor-associated macrophages have been reported to be related to poor prognosis in several human cancers [63]. However, contrary to the aforementioned roles of Ninj1 in modulating cell motility, Park et al. [64] found that the expression of Ninj1 was highly upregulated in circulating tumor cells derived from the blood of locally advanced prostate cancer patients, and Ninj1 overexpression could enhance prostate cancer cell migration and invasion in vitro.

Ninj1 Is Involved in Tumorigenesis in Other Ways

In addition to the roles discussed above, Ninj1 was also observed to be increased in hepatocellular carcinoma combined with viral infection and cirrhosis [7]. Furthermore, enforced Ninj1 expression in a human hepatoma cell line upregulated the expression of $\mathrm{p} 21$ by posttranscriptional induction, which has been reported to be crucial for the establishment of cellular senescence [65], thereby triggering a cellular response resulting in G1 cell cycle arrest and cellular senescence [66]. Interestingly, the role of Ninj1 regulating the expression of $\mathrm{p} 21$ is independent of p53 [66]. Furthermore, with respect to hematological cancers, the expression of Ninj1 is upregulated in $\mathrm{B}$-lineage acute lymphoblastic leukemia, and Ninj1 is also identified as a novel marker of minimal residual disease in the B-lineage acute lymphoblastic leukemia via cDNA microarrays [67]. Moreover, there are also some research reports which found that Ninj1 may also be a novel biomarker of nonmuscle-invasive urothelial bladder cancer 
prognosis $[68,69]$. Specifically, tumors highly expressing Ninj1 were inclined to exhibit a slower progression than those without expressing Ninj1 $[68,69]$.

\section{The Role of Ninjurin1 in Tissue Homeostasis}

Ninj1 Is Involved in Vascular Homeostasis

As we all know, it is well documented that angiogenesis, the formation of new capillary sprouting from the existing blood vessels, is considered as a crucial pathophysiological process of tissue development and various diseases, including wound healing, tumor progression, ischemia, and inflammation, not only during fetal development but also after birth [70, 71]. Angiopoietins, the specific ligands of the Tie 2 receptor tyrosine kinase, play a crucial role in angiogenesis, vascular remodeling, and maturation of blood vessels [72-74]. Among them, angiopoietin-1 (Ang1) is relevant to cell survival by activating the PI3K/Akt signaling pathway, whereas angiopoietin-2 (Ang2), an endogenous antagonist of Ang1, participates in inducing cell apoptosis via inhibiting the PI3K/Akt signaling pathway [75]. And, accumulating evidence has demonstrated that Ninj1 was involved in the vascular homeostasis of various pathophysiologic processes by regulating the expression of Ang1 and Ang2 [46, 76-79]. Firstly, Lee et al. [78] found that Ninj1 was temporally increased in the surface of macrophages, and transiently expressed Ninj1 contributed to the regression of the hyaloid vascular system, a transient existing capillary network participating in the maturation of lens during early ocular development, by enhancing the expression of Ang2 and Wnt7b, leading to the apoptosis of vascular ECs, and reducing the expression of Ang1. Moreover, Ninj1-neutralizing antibody delayed the regression of the hyaloid vascular system in vivo [78]. Thus, Ninj1 plays a vital role in vascular homeostasis during the embryonic stage.

Furthermore, Ninj1 is also considered as a novel therapeutic target for ED, caused by various pathogenetic factors, including endothelial dysfunction and peripheral neuropathy [79]. On the one hand, Yin et al. [45] demonstrated that the expression of Ninj1 was extremely upregulated both in cavernous ECs of diabetic mice with ED in vivo and primary cultured mouse cavernous ECs under the HG condition in vitro. Moreover, inhibition of Ninj1 could restore the erectile function of diabetic mice partly through promoting the proliferation and decreasing the apoptosis of cavernous ECs via the Ang1-Tie2 signal pathway [46]. Specifically, the expression of Ang1 was decreased, and the expression of Ang2 was increased in the diabetic penis, whereas Ninj1-neutralizing antibody could reverse this change and therefore enhance the cavernous angiogenesis and subsequently rescue erectile function [46]. On the other hand, Ninj1 is also increased in the penis in a mouse model of cavernous nerve injury (CNI), which is the main cause of radical prostatectomyinduced ED [79-81]. Furthermore, systemic neutralization with the Ninj1 antibody also could restore the erectile function of the CNI mice, at least partly, via regulating the expression of Ang1 and Ang2 [79].

Additionally, Ninj1 also could regulate vascular homeostasis through the function of pericytes (PCs) [76, 77], which participate in the whole process of angiogenesis via specific trophic functions and the dynamic interaction with ECs [82, 83]. Matsuki et al. [77] elucidated that Ninj1 was enhanced in capillary cells, especially in PCs, during hypoxia and inflammatory cytokine-induced angiogenesis in vitro. Furthermore, upregulated Ninj1 in PCs negatively regulates EC tube formation via restraining the trophic functions of PCs, which regulates the production of pro- and antiangiogenic factors, in especially Ang1 and Ang2 [77]. Therefore, according to the above research results, Ninj1 mainly acts as a negative regulator in the process of EC tube formation and elicits a crucial role in the regulation of vascular system homeostasis.

\section{Ninj1 Is Involved in Bone Homeostasis and Muscle}

Homeostasis

In addition to its role in vascular homeostasis [78], Ninj1 also plays a vital role in other tissue homeostasis. On the one hand, Bae et al. [84] demonstrated that Ninj1 was highly expressed in mature osteoclasts (OCs), which are myeloid lineage descendants, and changed dynamically with the differentiation process of OCs. Furthermore, Ninj1 could promote the survival of prefusion OCs (preOCs) via alleviating the caspase-9-dependent endogenous apoptosis of preOCs, thereby affecting the development of OCs and bone homeostasis [84]. Furthermore, high expression of NINJ1 is also relevant to destructive bone diseases, such as rheumatoid arthritis and osteoporosis, by microarray analysis [84]. Thus, they can conclude that Ninj1 has vital functions during bone homeostasis.

On the other hand, Kny et al. [85] disclosed that Ninj1 was expressed in cardiomyocytes and increased during pathological cardiac hypertrophy in both humans and mice. More importantly, Ninj1, especially N-glycosylated Ninj1, could impact muscle homeostasis via regulating myogenic differentiation and myocyte growth, while non-N-glycosylated Ninj 1 probably was associated with migration and fusion of myocytes [85]. Moreover, given 
that the NINJ1 gene is located at the chromosome 9q22.1q22.3 [1] and a possible disease site of familial dilated cardiomyopathy is located at the chromosome $9 \mathrm{q} 13-\mathrm{q} 22$ [86], NINJ1 is probably one of the causative genes of dilated cardiomyopathy. Therefore, Ninj1 plays an important role in muscle homeostasis as well.

\section{Discussion}

Ninj1 is a 2-pass membrane protein with CAM functions. In general, the functions of Ninj1 mainly cover the following aspects, including promoting peripheral nerve regeneration, promoting inflammation inside and outside the nervous system, and participating in the regulation of tumorigenesis as well as maintaining vascular, bone, and muscle homeostasis. However, with the deepening of research on the role of Ninj1, some studies have put forward different views on the role of Ninj1 in certain aspects. First of all, regarding the role of Ninj1 in promoting the growth of peripheral nerves, the research of Yin et al. [46] has reached different conclusions. They found that inhibition of Ninj1 could promote nerve regeneration in the mouse model of both DM-induced ED and CNI-induced ED $[46,79]$. These results imply that Ninj1 may play a harmful or protective role in an organ- or context-specific way in the nerve system.

Furthermore, as for the role of Ninj1 in regulating vascular homeostasis, Minoshima et al. [89] have different findings as well. According to previous research reports, they can conclude that Ninj1 can inhibit the formation of EC tubes by reducing the nutritional effect of PCs [77]. However, suppression of EC growth and migration by PCs may not always cause the regression of vessels and is also related to vascular maturation and stabilization to form functional vessels $[87,88]$. Thus, Minoshima et al. constructed a hindlimb ischemia model by using WT and PC-specific Ninj1-knockout murine. And, they disclosed that Ninj1 in PCs could promote the formation of functional vessels within the ischemic tissues, thus could promote the blood flow recovery in hindlimb ischemia via the association between PCs and ECs [89]. Intriguingly, the expression of Ninj1 is upregulated in both ischemic skeletal muscle tissue and penile tissue under diabetic conditions, whereas its role in regulating vascular homeostasis is contradictory in both states. One possible explanation for this phenomenon is that the condition of Ninj1 expression is different, that is, long-term chronic increased Ninj1 under DM condition versus temporal acute increased Ninj1 in ischemic tissues [89]. More im- portant, strategies to inhibit the expression and function of Ninj1 would be therapeutic in some cases, for instance, CNI-induced ED and DM-induced ED [46, 79]. However, functional inhibition of Ninj1 may also lead to adverse effects in certain conditions, such as ischemia [89].

Moreover, it has been proved that Ninj1 is induced to increase during inflammation in multiple inflammatory models and plays a proinflammatory role in a variety of ways. Intriguingly, Choi et al. [90] reached the opposite conclusion about the role of Ninj1 in intestinal inflammation. They found that Ninj1 can alleviate the development of severe colitis by regulating the M1/M2 polarization of macrophages and maintaining the homeostasis of intestinal microbes [90]. Both the polarized phenotype of macrophages and intestinal microbial balance are believed to be closely related to the occurrence and progression of intestinal inflammatory diseases [90-92]. Specifically, via reducing the proportion of proinflammatory M1 macrophages and increasing the proportion of antiinflammatory M2 macrophages, Ninj1 can mitigate the lesion of colitis and facilitate the recovery of colitis during the progression of colitis [90]. Therefore, the role of Ninj1 in intestinal inflammation needs more extensive and indepth researches. Besides, as a novel therapeutic target of inflammation, Ninj1 is more anticipated than other CAMs, such as very late antigen-4 (VLA-4) and lymphocyte function-associated molecule-1 (LFA-1). After the CNS is stimulated by inflammation, VLA-4 and LFA-1 are highly expressed in most immune cell subtypes including monocytes and T lymphocytes [93, 94], while Ninj1 is cell specific and weakly expressed in noninflamed areas [26]. Thus, although the application of VLA-4 and LFA-1 blockers can achieve a good therapeutic effect by reducing the infiltration of leukocytes into the target organs [95-97], these widespread targeting inhibition methods also affect the immune system so that it cannot maintain antibacterial, antivirus, and antitumor immune surveillance functions. Actually, it has been reported that patients treated with these CAM inhibitors experience relatively high frequency and intensity of adverse reactions and even life-threatening complications, including progressive multifocal leukoencephalopathy and rapidly progressing malignant melanoma $[98,99]$. Nevertheless, blocking Ninj1 is likely to achieve an effective therapeutic effect without altering the critical baseline immunosurveillance of the immune system.

According to the research of Choi et al. [90], Ninj1 could be induced after peripheral tissue ischemia and facilitate blood flow recovery by promoting the formation of functional blood vessels [89]. However, the vascular 
regulation effect of Ninj1 in ischemic cerebrovascular disease has not been studied. Although the Ninj1 blockade could exert powerful anti-inflammatory and neuroprotective effects by inhibiting the Ninj1-induced infiltration of neutrophils into the brain in the rat MCAO model [30], we still believe that the vascular regulation effect of Ninj1 in ischemic cerebrovascular disease is worth studying. Because this may provide further evidence for the view that Ninj1 is a new therapeutic target for ischemic stroke, we are currently engaged in related research. In addition, although there has been a large amount of literature proving that Ninj1 is involved in a variety of pathophysiological processes in the body, the specific molecular mechanism of Ninj1 in these processes still needs much deeper research. Moreover, most of the reported studies on Ninj1 are still at the level of cell and animal research. We believe that the next step in research on Ninj1 should be closer to the clinic, so that the results of basic research can be transformed into clinically usable treatment options as soon as possible.

\section{Conclusion}

In general, Ninj1 is widely involved in many pathophysiological processes in the body, including promoting nerve regeneration, facilitating inflammation, regulating tumorigenesis, and maintaining vascular, bone, and muscle homeostasis. Although the current mechanism of Ninj1 in these pathophysiological processes is still not very clarified, even some conclusions are contradictory. Ninj1 is indeed considered as a new therapeutic target in certain diseases, such as ED. More extensive and in-depth research of Ninj1 is very meaningful and worth looking forward to.

\section{Conflict of Interest Statement}

The other authors declare no competing interests.

\section{Funding Sources}

This work was supported by grants from the National Natural Science Foundation of China (No. 81801181).

\section{Author Contributions}

Ke Liu found the relevant research basis and wrote this article. Hongge Li and Yong Wang designed the review and revised the article. All authors read and approved the final version of the manuscript.

\section{References}

1 Araki T, Milbrandt J. Ninjurin, a novel adhesion molecule, is induced by nerve injury and promotes axonal growth. Neuron. 1996; 17(2):353-61.

2 Araki T, Zimonjic DB, Popescu NC, Milbrandt J. Mechanism of homophilic binding mediated by ninjurin, a novel widely expressed adhesion molecule. J Biol Chem. 1997;272(34):21373-80.

3 Lee HJ, Ahn BJ, Shin MW, Choi JH, Kim KW. Ninjurin1: a potential adhesion molecule and its role in inflammation and tissue remodeling. Mol Cells. 2010;29(3):223-7.

4 Araki T, Milbrandt J. Ninjurin2, a novel homophilic adhesion molecule, is expressed in mature sensory and enteric neurons and promotes neurite outgrowth. J Neurosci. 2000; 20(1):187-95.

5 Bae SJ, Shin MW, Kim RH, Shin D, Son T, Wee HJ, et al. Ninjurin 1 assembles into a homomeric protein complex maintained by $\mathrm{N}$ linked glycosylation. J Cell Biochem. 2017; 118(8):2219-30.

6 Jakovcevski I, Wu J, Karl N, Leshchyns'Ka I, Sytnyk V, Chen J, et al. Glial scar expression of chll, the close homolog of the adhesion molecule 11, limits recovery after spinal cord injury. J Neurosci. 2007;27(27):7222-33.

7 Kim JW, Moon AR, Kim JH, Yoon SY, Oh GT, Choe YK, et al. Up-regulation of ninjurin expression in human hepatocellular carcinoma associated with cirrhosis and chronic viral hepatitis. Mol Cells. 2001;11(2):151.

8 Schlosshauer B, Schwarz U, Rutishauser U. Topological distribution of different forms of neural cell adhesion molecule in the developing chick visual system. Nature. 1984; 310(5973):141-3.

9 Menorca RM, Fussell TS, Elfar JC. Nerve physiology: mechanisms of injury and recovery. Hand Clin. 2013;29(3):317-30.

10 Wong KM, Babetto E, Beirowski B. Axon degeneration: make the Schwann cell great again. Neural Regen Res. 2017;12(4):51824.

11 Kubo T, Yamashita T, Yamaguchi A, Hosokawa $\mathrm{K}$, Tohyama M. Analysis of genes induced in peripheral nerve after axotomy using cDNA microarrays. J Neurochem. 2002; 82(5):1129-36.

12 Lemmon V, Farr KL, Lagenaur C. L1-mediated axon outgrowth occurs via a homophilic binding mechanism. Neuron. 1989;2(6): 1597-603.

13 Takeichi M. Cadherin cell adhesion receptors as a morphogenetic regulator. Science. 1991; 251(5000): 1451-5.

14 Jerregard H. Sensory neurons influence the expression of cell adhesion factors by cutaneous cells in vitro and in vivo. J Neurocytol. 2001;30(4):327-36.

15 Tomita Y, Horiuchi K, Kano K, Tatsukawa T, Matsuo R, Hayasaka T, et al. Ninjurin 1 mediates peripheral nerve regeneration through Schwann cell maturation of NG2-positive cells. Biochem Biophys Res Commun. 2019; 519(3):462-8.

16 Rambukkana A. Usage of signaling in neurodegeneration and regeneration of peripheral nerves by leprosy bacteria. Prog Neurobiol. 2010;91(2):102-7.

17 Ooi WW, Srinivasan J. Leprosy and the peripheral nervous system: basic and clinical aspects. Muscle Nerve. 2004;30(4):393-409.

18 Agrawal A, Pandit L, Dalal M, Shetty JP. Neurological manifestations of Hansen's disease and their management. Clin Neurol Neurosurg. 2005;107(6):445-54. 
19 Cardoso CC, Martinez AN, Guimarães PE, Mendes CT, Pacheco AG, de Oliveira RB, et al. Ninjurin 1 asp110ala single nucleotide polymorphism is associated with protection in leprosy nerve damage. J Neuroimmunol. 2007;190(1-2):131-8.

20 Graca CR, Paschoal VD, Cordeiro-Soubhia RM, Tonelli-Nardi SM, Machado RL, Kouyoumdjian JA, et al. Ninjurin1 single nucleotide polymorphism and nerve damage in leprosy. Infect Genet Evol. 2012;12:597-600.

21 Miyashita M, Koga K, Takeuchi A, Makabe T, Taguchi A, Urata Y, et al. Expression of nerve injury-induced protein1 (ninj1) in endometriosis. Reprod Sci. 2019;26(8):1105-10.

22 Ahn BJ, Lee HJ, Shin MW, Choi JH, Jeong JW, Kim KW. Ninjurin 1 is expressed in myeloid cells and mediates endothelium adhesion in the brains of EAE rats. Biochem Biophys Res Commun. 2009;387(2):321-5.

23 Ransohoff RM, Kivisäkk P, Kidd G. Three or more routes for leukocyte migration into the central nervous system. Nat Rev Immunol. 2003;3(7):569-81.

24 Ley K, Laudanna C, Cybulsky MI, Nourshargh S. Getting to the site of inflammation: the leukocyte adhesion cascade updated. Nat Rev Immunol. 2007;7(9):678-89.

25 Ahn BJ, Le H, Shin MW, Bae SJ, Lee EJ, Wee $\mathrm{HJ}$, et al. Ninjurin 1 deficiency attenuates susceptibility of experimental autoimmune encephalomyelitis in mice. J Biol Chem. 2014; 289(6):3328-38.

26 Ifergan I, Kebir H, Terouz S, Alvarez JI, Lécuyer MA, Gendron S, et al. Role of ninjurin-1 in the migration of myeloid cells to central nervous system inflammatory lesions. Ann Neurol. 2011;70(5):751-63.

27 Odoardi F, Sie C, Streyl K, Ulaganathan VK, Schläger C, Lodygin $\mathrm{D}$, et al. T cells become licensed in the lung to enter the central nervous system. Nature. 2012;488(7413):675-9.

28 Lee HK, Lee H, Luo L, Lee JK. Induction of nerve injury-induced protein 1 (ninjurin 1 ) in myeloid cells in rat brain after transient focal cerebral ischemia. Exp Neurobiol. 2016;25(2): 64-74.

29 Ahn BJ, Le H, Shin MW, Bae SJ, Lee EJ, Lee SY, et al. Ninjurin1 enhances the basal motility and transendothelial migration of immune cells by inducing protrusive membrane dynamics. J Biol Chem. 2014;289(32):21926-36.

30 Lee HK, Kim ID, Lee H, Luo L, Kim SW, Lee JK. Neuroprotective and anti-inflammatory effects of a dodecamer peptide harboring ninjurin 1 cell adhesion motif in the postischemic brain. Mol Neurobiol. 2018;55(7):6094-111.

31 Le H, Ahn BJ, Lee HS, Shin A, Chae S, Lee SY, et al. Disruption of ninjurin1 leads to repetitive and anxiety-like behaviors in mice. Mol Neurobiol. 2017;54(9):7353-68.

32 Jennewein C, Sowa R, Faber AC, Dildey M, von Knethen A, Meybohm P, et al. Contribution of ninjurin 1 to Toll-like receptor 4 signaling and systemic inflammation. Am J Respir Cell Mol Biol. 2015;53(5):656-63.
33 Shin MW, Bae SJ, Wee HJ, Lee HJ, Ahn BJ, Le $\mathrm{H}$, et al. Ninjurin1 regulates lipopolysaccharide-induced inflammation through direct binding. Int J Oncol. 2016;48(2):821-8.

34 Tamoutounour S, Henri S, Lelouard H, de Bovis $B$, de Haar C, van der Woude CJ, et al. CD64 distinguishes macrophages from dendritic cells in the gut and reveals the Th1-inducing role of mesenteric lymph node macrophages during colitis. Eur J Immunol. 2012; 42(12):3150-66.

35 Wang S, Ye Q, Zeng X, Qiao S. Functions of macrophages in the maintenance of intestinal homeostasis. J Immunol Res. 2019;2019: 1512969.

36 Jung HJ, Kang J-H, Pak S, Lee K, Seong JK, Oh $\mathrm{SH}$. Detrimental role of nerve injury-induced protein 1 in myeloid cells under intestinal inflammatory conditions. Ijms. 2020;21(2):614.

37 Borthwick LA, Barron L, Hart KM, Vannella KM, Thompson RW, Oland S, et al. Macrophages are critical to the maintenance of il13-dependent lung inflammation and fibrosis. Mucosal Immunol. 2016;9(1):38-55.

38 Wynn TA. Cellular and molecular mechanisms of fibrosis. J Pathol. 2008;214(2):199210.

39 Bringardner BD, Baran CP, Eubank TD, Marsh CB. The role of inflammation in the pathogenesis of idiopathic pulmonary fibrosis. Antioxid Redox Signal. 2008;10(2):287301.

40 Selman M, King TE, Pardo A. Idiopathic pulmonary fibrosis: prevailing and evolving hypotheses about its pathogenesis and implications for therapy. Ann Intern Med. 2001; 134(2):136-51.

41 King TE, Pardo A, Selman M. Idiopathic pulmonary fibrosis. Lancet. 2011;378(9807): 1949-61.

42 Choi S, Woo JK, Jang Y-S, Kang J-H, Hwang J-I, Seong JK, et al. Ninjurin 1 plays a crucial role in pulmonary fibrosis by promoting interaction between macrophages and alveolar epithelial cells. Sci Rep. 2018;8(1):17542.

43 Zhang L, Zalewski A, Liu Y, Mazurek T, Cowan S, Martin JL, et al. Diabetes-induced oxidative stress and low-grade inflammation in porcine coronary arteries. Circulation. 2003; 108(4):472-8.

44 Wang X, Qin J, Zhang X, Peng Z, Ye K, Wu X, et al. Functional blocking of ninjurin1 as a strategy for protecting endothelial cells in diabetes mellitus. Clin Sci. 2018;132(2):213-29.

45 Kim DK, Yin GN, Ryu JK, Suh JK. Differential expression of nerve injury-induced protein 1 (ninjurin 1) in in vivo and in vitro models for diabetic erectile dysfunction. Korean J Urol. 2012;53(9):636.

46 Yin GN, Choi MJ, Kim WJ, Kwon MH, Song KM, Park JM, et al. Inhibition of ninjurin 1 restores erectile function through dual angiogenic and neurotrophic effects in the diabetic mouse. Proc Natl Acad Sci USA. 2014; 111(26):E2731-40.
47 Bieging KT, Attardi LD. Deconstructing p53 transcriptional networks in tumor suppression. Trends Cell Biol. 2012;22(2):97-106.

48 Vogelstein B, Lane D, Levine AJ. Surfing the p53 network. Nature. 2000;408(6810):30710.

49 Vousden KH, Prives C. Blinded by the light: the growing complexity of p53. Cell. 2009; 137(3):413-31.

50 Kannan K, Amariglio N, Rechavi G, JakobHirsch J, Kela I, Kaminski N, et al. DNA microarrays identification of primary and secondary target genes regulated by $\mathrm{p} 53$. Oncogene. 2001;20(18):2225-34.

51 Cho SJ, Rossi A, Jung YS, Yan W, Liu G, Zhang J, et al. Ninjurin1, a target of p53, regulates p53 expression and p53-dependent cell survival, senescence, and radiation-induced mortality. Proc Natl Acad Sci USA. 2013; 110(23):9362-7.

52 Komarova EA, Kondratov RV, Wang K, Christov K, Golovkina TV, Goldblum JR, et al. Dual effect of p53 on radiation sensitivity in vivo: 553 promotes hematopoietic injury, but protects from gastro-intestinal syndrome in mice. Oncogene. 2004;23(19): 3265-71.

53 Koike M, Ninomiya Y, Koike A. Characterization of ninjurin and TSC22 induction after Xirradiation of normal human skin cells. J Dermatol. 2008;35(1):6-17.

54 Yang HJ, Zhang J, Yan W, Cho SJ, Lucchesi C, Chen M, et al. Ninjurin 1 has two opposing functions in tumorigenesis in a p53-dependent manner. Proc Natl Acad Sci USA. 2017; 114(43):11500-5.

55 Jang YS, Kang JH, Woo JK, Kim HM, Hwang JI, Lee SJ, et al. Ninjurin1 suppresses metastatic property of lung cancer cells through inhibition of interleukin 6 signaling pathway. Int J Cancer. 2016;139(2):383-95.

56 Kesanakurti D, Chetty C, Rajasekhar Maddirela D, Gujrati M, Rao JS. Essential role of cooperative NF- $\mathrm{kB}$ and Stat3 recruitment to ICAM-1 intronic consensus elements in the regulation of radiation-induced invasion and migration in glioma. Oncogene. 2013;32(43): 5144-55.

57 Huang WC, Chan ST, Yang TL, Tzeng CC, Chen CC. Inhibition of ICAM-1 gene expression, monocyte adhesion and cancer cell invasion by targeting IKK complex: molecular and functional study of novel alpha-methylenegamma-butyrolactone derivatives. Carcinogenesis. 2004;25(10):1925-34.

58 Lalancette M, Aoudjit F, Potworowski EF, StPierre Y. Resistance of ICAM-1-deficient mice to metastasis overcome by increased aggressiveness of lymphoma cells. Blood. 2000; 95(1):314-9.

59 Maruo Y, Gochi A, Kaihara A, Shimamura H, Yamada T, Tanaka N, et al. ICAM-1 expression and the soluble ICAM-1 level for evaluating the metastatic potential of gastric cancer. Int J Cancer. 2002;100(4):486-90. 
60 O'Hanlon DM, Fitzsimons H, Lynch J, Tormey S, Malone C, Given HF. Soluble adhesion molecules (E-selectin, ICAM-1 and VCAM-1) in breast carcinoma. Eur J Cancer. 2002;38(17):2252-7.

61 Tempia-Caliera AA, Horvath LZ, Zimmermann A, Tihanyi TT, Korc M, Friess H, et al. Adhesion molecules in human pancreatic cancer. J Surg Oncol. 2002;79(2):93-100.

62 Woo JK, Jang YS, Kang JH, Hwang JI, Seong JK, Lee SJ, et al. Ninjurin1 inhibits colitis-mediated colon cancer development and growth by suppression of macrophage infiltration through repression of FAK signaling. Oncotarget. 2016;7(20):29592-604.

63 Bingle L, Brown NJ, Lewis CE. The role of tumour-associated macrophages in tumour progression: implications for new anticancer therapies. J Pathol. 2002;196(3):254-65.

64 Park J, Joung JY, Hwang JE, Hong D, Park WS, Lee SJ, et al. Ninjurin1 is up-regulated in circulating prostate tumor cells and plays a critical role in prostate cancer cell motility. Anticancer Res. 2017;37(4):1687-96.

65 Burkhart BA, Alcorta DA, Chiao C, Isaacs JS, Barrett JC. Two posttranscriptional pathways that regulate $\mathrm{p} 21$ (Cip1/Waf1/Sdi1) are identified by HPV16-E6 interaction and correlate with life span and cellular senescence. Exp Cell Res. 1999;247(1):168-75.

66 Toyama T, Sasaki Y, Horimoto M, Iyoda K, Yakushijin T, Ohkawa K, et al. Ninjurin1 increases p21 expression and induces cellular senescence in human hepatoma cells. J Hepatol. 2004;41(4):637-43.

67 Chen JS, Coustan-Smith E, Suzuki T, Neale GA, Mihara K, Pui CH, et al. Identification of novel markers for monitoring minimal residual disease in acute lymphoblastic leukemia. Blood. 2001 Apr 1;97(7):2115-20.

68 Mhawech-Fauceglia P, Ali L, Cheney RT, Groth J, Herrmann FR. Prognostic significance of neuron-associated protein expression in non-muscle-invasive urothelial bladder cancer. J Clin Pathol. 2009;62(8):710-4.

69 Mares J, Szakacsova M, Soukup V, Duskova J, Horinek A, Babjuk M. Prediction of recurrence in low and intermediate risk non-muscle invasive bladder cancer by real-time quantitative PCR analysis: cDNA microarray results. Neoplasma. 2013;60(3):295-301.

70 Folkman J. Angiogenesis: an organizing principle for drug discovery? Nat Rev Drug Discov. 2007;6(4):273-86.

71 Potente M, Gerhardt H, Carmeliet P. Basic and therapeutic aspects of angiogenesis. Cell. 2011;146(6):873-87.

72 Davis S, Aldrich TH, Jones PF, Acheson A, Compton DL, Jain V, et al. Isolation of angiopoietin-1, a ligand for the Tie2 receptor, by secretion-trap expression cloning. Cell. 1996; 87(7):1161-9.
73 Ward NL, Dumont DJ. The angiopoietins and Tie2/TEK: adding to the complexity of cardiovascular development. Semin Cell Dev Biol. 2002;13(1):19-27.

74 Suri C, Jones PF, Patan S, Bartunkova S, Maisonpierre PC, Davis S, et al. Requisite role of angiopoietin-1, a ligand for the Tie2 receptor, during embryonic angiogenesis. Cell. 1996; 87(7):1171-80.

75 Augustin HG, Koh GY, Thurston G, Alitalo K. Control of vascular morphogenesis and homeostasis through the angiopoietin-Tie system. Nat Rev Mol Cell Biol. 2009;10(3): 165-77.

76 Fujita Y, Kawamoto A. Ninjurin1 - a novel regulator of angiogenesis mediated by pericytes. Circ J. 2015;79(6):1218-9.

77 Matsuki M, Kabara M, Saito Y, Shimamura K, Minoshima A, Nishimura M, et al. Ninjurin1 is a novel factor to regulate angiogenesis through the function of pericytes. Circ J. 2015; 79(6):1363-71.

78 Lee HJ, Ahn BJ, Shin MW, Jeong JW, Kim JH, Kim KW. Ninjurin1 mediates macrophageinduced programmed cell death during early ocular development. Cell Death Differ. 2009; 16(10):1395-407.

79 Yin GN, Kim WJ, Jin HR, Kwon MH, Song KM, Choi MJ, et al. Nerve injury-induced protein 1 (ninjurin-1) is a novel therapeutic target for cavernous nerve injury-induced erectile dysfunction in mice. J Sex Med. 2013; 10(6):1488-501.

80 Walsh PC, Mostwin JL. Radical prostatectomy and cystoprostatectomy with preservation of potency. Results using a new nerve-sparing technique. Br J Urol. 1984;56(6):694-7.

81 Noldus J, Michl U, Graefen M, Haese A, Hammerer P, Huland H. Patient-reported sexual function after nerve-sparing radical retropubic prostatectomy. Eur Urol. 2002; 42(2):118-24.

82 Diaz-Flores L, Gutierrez R, Madrid JF, Varela H, Valladares F, Acosta E, et al. Pericytes. Morphofunction, interactions and pathology in a quiescent and activated mesenchymal cell niche. Histol Histopathol. 2009;24:909-69.

83 Armulik A, Genové G, Betsholtz C. Pericytes: developmental, physiological, and pathological perspectives, problems, and promises. Dev Cell. 2011;21(2):193-215.

84 Bae S, Shin MW, Son T, Lee HS, Chae JS, Jeon $\mathrm{S}$, et al. Ninjurin1 positively regulates osteoclast development by enhancing the survival of prefusion osteoclasts. Exp Mol Med. 2019; 51:1-15.

85 Kny M, Csályi KD, Klaeske K, Busch K, Meyer AM, Merks AM, et al. Ninjurin1 regulates striated muscle growth and differentiation. Plos One. 2019;14(5):e0216987.
86 Krajinovic M, Pinamonti B, Sinagra G, Vatta M, Severini GM, Milasin J, et al. Linkage of familial dilated cardiomyopathy to chromosome 9. Heart muscle disease study group. Am J Hum Genet. 1995;57(4):846-52.

87 Jain RK. Molecular regulation of vessel maturation. Nat Med. 2003;9(6):685-93.

88 Armulik A, Abramsson A, Betsholtz C. Endothelial/pericyte interactions. Circ Res. 2005; 97(6):512-23.

89 Minoshima A, Kabara M, Matsuki M, Yoshida Y, Kano K, Tomita Y, et al. Pericyte-specific ninjurin 1 deletion attenuates vessel maturation and blood flow recovery in hind limb ischemia. Arterioscler Thromb Vasc Biol. 2018;38(10):2358-70.

90 Choi H, Bae SJ, Choi G, Lee H, Son T, Kim JG, et al. Ninjurin1 deficiency aggravates colitis development by promoting $\mathrm{m} 1$ macrophage polarization and inducing microbial imbalance. Faseb J. 2020;34(6):8702-20.

$91 \mathrm{Na}$ YR, Stakenborg M, Seok SH, Matteoli G. Macrophages in intestinal inflammation and resolution: a potential therapeutic target in IBD. Nat Rev Gastroenterol Hepatol. 2019; 16(9):531-43.

92 Levy M, Kolodziejczyk AA, Thaiss CA, Elinav E. Dysbiosis and the immune system. Nat Rev Immunol. 2017;17(4):219-32.

93 Kebir H, Ifergan I, Alvarez JI, Bernard M, Poirier J, Arbour N, et al. Preferential recruitment of interferon-gamma-expressing th 17 cells in multiple sclerosis. Ann Neurol. 2009; 66(3):390-402.

94 Archelos JJ, Previtali SC, Hartung HP. The role of integrins in immune-mediated diseases of the nervous system. Trends Neurosci. 1999;22(1):30-8.

95 Miller DH, Khan OA, Sheremata WA, Blumhardt LD, Rice GP, Libonati MA, et al. A controlled trial of natalizumab for relapsing multiple sclerosis. N Engl J Med. 2003;348(1): $15-23$.

96 Lebwohl M, Tyring SK, Hamilton TK, Toth D, Glazer S, Tawfik NH, et al. A novel targeted T-cell modulator, efalizumab, for plaque psoriasis. N Engl J Med. 2003;349(21): 2004-13.

97 Ghosh S, Goldin E, Gordon FH, Malchow HA, Rask-Madsen J, Rutgeerts P, et al. Natalizumab for active Crohn's disease. N Engl Med. 2003;348(1):24-32.

98 Langer-Gould A, Atlas SW, Green AJ, Bollen AW, Pelletier D. Progressive multifocal leukoencephalopathy in a patient treated with natalizumab. N Engl J Med. 2005 Jul 28; 353(4):375-81.

99 Mullen JT, Vartanian TK, Atkins MB. Melanoma complicating treatment with natalizumab for multiple sclerosis. N Engl J Med. 2008;358(6):647-8. 